\title{
Vibration Analysis and Stability Analysis of Double-Walled Carbon Nanotubes Subjected to Electrostatics Actuation
}

\author{
Tai-Ping Chang* \\ Department of Construction Engineering, National Kaohsiung First University of Science and Technology, \\ 1 University road, Yanchao, Kaohsiung 824, Taiwan \\ (Received March 22, 2016; accepted May 10, 2017)
}

Keywords: double-walled carbon nanotubes, vibration response, instability behavior, electrostatic actuation, stochastic modeling

In this study, the nonlinear vibration response and instability behavior of double-walled carbon nanotubes (DWCNTs) subjected to electrostatic actuation are investigated. Both deterministic and stochastic modeling are considered. In deterministic modeling, two Euler-Bernoulli beams are adopted to model the inner and outer layers of the DWCNTs. The governing equations of motion are solved by Galerkin and multiple scales methods for primary and secondary resonances. The frequency response of the system is obtained as a function of some of the system parameters. The stability analysis of the response is performed and bifurcation points are obtained. In stochastic modeling, the finite element formulation and the perturbation technique are adopted to investigate the vibration response and instability behavior of the DWCNTs under electrostatic actuation. Note that both layers of the DWCNTs vibrate with the same frequency under the primary and secondary resonance conditions. The nonlinear vibration behaviors in both deterministic and stochastic cases are very similar to each other, except in the stochastic case, some statistical quantities such as the mean values and standard deviations of the displacements of DWCNTs must be considered and dealt with in the whole process.

\section{Introduction}

Recently, research on static mechanical properties and dynamic behaviors of nanotubes has been well established. The applications of double-walled carbon nanotubes (DWCNTs) in vibrating devices and the nonlinear nature of the $\mathrm{vdW}$ (van der Waals) forces and electrical forces strongly demand a comprehensive study of DWCNT dynamic properties, especially under external excitations such as vibrations and electric actuations. Some researchers ${ }^{(1-7)}$ have made contributions to the study of the static and dynamic behaviors of nanotubes by modelling each layer of the nanotubes as Euler-Bernoulli or Timoshenko beams. Dequesnes et al. ${ }^{(8)}$ adopted the EulerBernoulli beam theory with a stretching term to investigate nanotubes vibrating under nonlinear $\mathrm{vdW}$ and electrostatic forces. The resulting equations were solved using the finite cloud method. They also dealt with the pull-in instability of nanotubes under DC voltage. Ouakad and Younis ${ }^{(9)}$ studied the nonlinear dynamics of clamped-clamped and cantilevered CNTs under DC and AC electrical excitation. Similar studies were conducted on microbeams. ${ }^{(10-12)}$ Hawwa and Al- 
Qahtani ${ }^{(13)}$ simplified the electric forces acting on a DWCNTs under harmonic electric force with a harmonic force acting on only the outer tube. In this study, forced vibrations of clamped-clamped DWCNTs under axial force and AC-DC complex electrostatic actuations are investigated using nonlinear Euler-Bernoulli beam theory. The stretching term is considered in the model and the nonlinear inertia term is neglected. The nonlinear interlayer vdW force is substituted by its Taylor expansion of nonlinear interlayer $\mathrm{vdW}$ force including its linear term and only the first nonlinear term. The multiple scales perturbation method is adopted to solve the dynamic response to a harmonic AC voltage. The steady-state responses of the layers of the DWCNTs under AC voltage with frequencies close to its natural frequencies and twice its natural frequencies are derived. The stability of the solutions and the stability boundaries of the system are studied. Finally, the stochastic modeling of the system is considered, namely, the Young's modulus of elasticity, and the mass distribution of the DWCNTs is characterized as a random field along the $x$-axis of the beam model to actually model the random properties of the DWCNTs. In addition, the finite element modeling of carbon nanotubes is adopted to carry out the stochastic analysis.

\section{Deterministic Modeling for DWCNTs under Electrostatic Actuation}

In Fig. 1, DWCNTs are characterized as a beam with a hollow circular cross section. The length of the DWCNTs is $L$, the external radius is represented by $R_{2}$, and $h$ indicates the effective tube thickness; the internal radius $R_{1}$ is not represented in the figure; the letter $d$ denotes the distance between the electrode and the DWCNTs in the undeformed state, also known as the initial gap width or the undeformed gap space. In this study, the Euler-Bernoulli beam theory is used to characterize the DWCNTs. The nonlinear vdW force between the DWCNT layers is also considered. The vibrations of the layers of the DWCNTs are coupled due to nonlinear forces. The clamped-clamped boundary conditions are considered for both layers. Therefore, considering the electrostatic force between the DWCNTs and the lower electrode, the equations of motion of the system are written as

$$
\begin{aligned}
E I_{1} \frac{\partial^{4} w_{1}}{\partial x^{4}}+\rho A_{1} \frac{\partial^{2} w_{1}}{\partial t^{2}} & +\xi_{1} \frac{\partial w_{1}}{\partial t}-\left(N_{1}+\frac{E A_{1}}{2 L} \int_{0}^{L}\left(\frac{\partial w_{1}}{\partial x}\right)^{2} d x\right) \frac{\partial^{2} w_{1}}{\partial x^{2}} \\
= & c_{3}\left[w_{2}-w_{1}\right]^{3}+c_{1}\left[w_{2}-w_{1}\right]+c_{s} q_{e}\left(w_{1}\right) V^{2}
\end{aligned}
$$

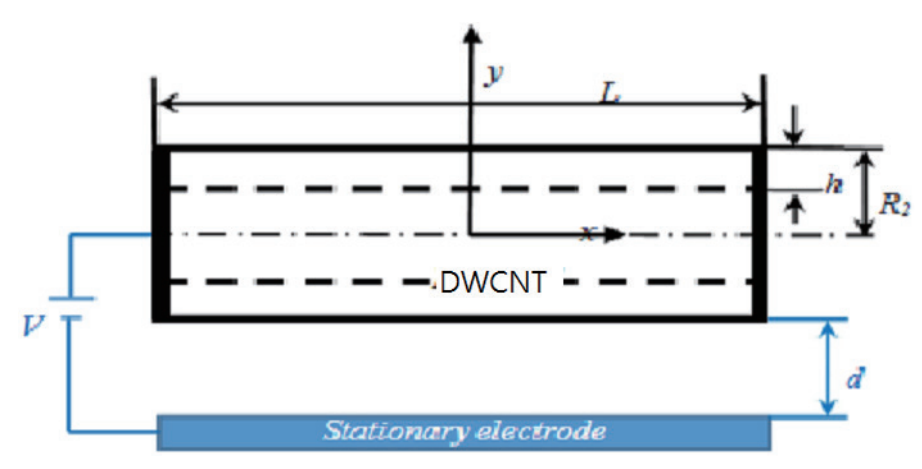

Fig. 1. (Color online) Electrostatic actuation of DWCNTs under clamped-clamped boundary conditions. 


$$
\begin{array}{r}
E I_{2} \frac{\partial^{4} w_{2}}{\partial x^{4}}+\rho A_{2} \frac{\partial^{2} w_{2}}{\partial t^{2}} \\
+\xi_{2} \frac{\partial w_{2}}{\partial t}-\left(N_{2}+\frac{E A_{2}}{2 L} \int_{0}^{L}\left(\frac{\partial w_{2}}{\partial x}\right)^{2} d x\right) \frac{\partial^{2} w_{2}}{\partial x^{2}} \\
=-c_{3}\left[w_{2}-w_{1}\right]^{3}-c_{1}\left[w_{2}-w_{1}\right]+q_{e}\left(w_{2}\right) V^{2}
\end{array}
$$

where $w_{1}$ and $w_{2}$ are the displacements of the inner and outer layers, respectively. $N_{1}$ and $N_{2}$ are the axial loads for the inner and outer tubes separately, and $c_{1}$ and $c_{3}$ are constant coefficients of the vdW forces whose numerical values are $c_{1}=71.11 \mathrm{GPa}$ and $c_{3}=2.57 \times 10^{4} \mathrm{GPa} \mathrm{nm}^{2}$. (7) The terms $\xi_{1}$ and $\xi_{2}$ are the damping coefficients of the inner and outer tubes, respectively, and $q_{e}\left(w_{n}\right)$ $V^{2}$ is the electrostatic force, which is applied between the layers of the DWCNTs and the lower electrode $^{(8)}$ In this forcing term, $V$ is the applied voltage, which is a combination of both $\mathrm{AC}$ and DC parts. The AC part is considered to be harmonic, and $c_{s}$ is the shielding effect constant, denoted here as the ratio of the applied electrostatic force on the inner tube when it is nested in the outer tube to the condition where the outer tube is removed. The total deflection of the DWCNTs is composed of a static part, $w_{s n}(x, t)$, and a dynamic part, $y_{n}(x, t)$, where $n=1,2 .^{(10-12,14,15)}$ The static and dynamic solutions are determined by the DC and AC voltages, respectively. To find the dynamic part, the AC and DC voltage components $\left(V=V_{d c}+V_{a c} \cos (\Omega t)\right)$ must be entered into Eqs. (1) and (2). Then, the electric force is substituted by their Taylor expansions. After that, static equations are subtracted from the resulting equations. To balance the nonlinearity with damping and excitation, they are considered to be of orders $O\left(y^{2}\right)$ and $O\left(y^{3}\right)$, respectively.

Finally, by removing the terms of orders higher than $O\left(y^{3}\right)$, the following equations are obtained:

$$
\begin{gathered}
\frac{\partial^{2} y_{1}(x, t)}{\partial t^{2}}-N\left(\frac{\partial^{2} y_{1}(x, t)}{\partial x^{2}}\right)+\frac{\partial^{4} y_{1}(x, t)}{\partial x_{4}} \\
-c_{1}\left(y_{2}(x, t)-y_{1}(x, t)\right)-3 \alpha_{2} c_{3} y_{2}(x, t)\left(w_{s 2}^{2}(x)+w_{s 1}^{2}(x)\right) \\
+3 c_{3} y_{1}(x, t)\left(w_{s 2}^{2}(x)+w_{s 1}^{2}(x)\right)+3 \alpha_{2} c_{3} y_{1}(x, t) y_{2}(x, t)\left(y_{1}(x, t)-y_{2}(x, t)\right) \\
+3 c_{3}\left(w_{s 1}(x)-w_{s 2}(x)\right)\left(y_{1}^{2}(x, t)-y_{2}^{2}(x, t)\right) \\
-2 c_{s} \alpha_{5} V_{a c} V_{d c}\left(e_{01}+e_{11} y_{2}(x, t)+e_{21} y_{1}^{2}(x, t)+e_{31} y_{1}^{3}(x, t)\right) \cos (\Omega t) \\
-c_{s} \alpha_{5} V_{d c}^{2}\left(e_{11} y_{1}(x, t)+e_{21} y_{1}^{2}(x, t)+e_{31} y_{1}^{3}(x, t)\right) \\
+\mu_{1}\left(\frac{\partial y_{1}(x, t)}{\partial t}\right)-6 c_{3} y_{1}(x, t) y_{2}(x, t)\left(w_{s 2}(x)-w_{s 1}(x)\right) \\
+6 c_{3} w_{s 2}(x) w_{s 1}(x)\left(y_{2}(x, t)-y_{1}(x, t)\right)-c_{3}\left(y_{2}^{3}(x, t)-y_{1}^{3}(x, t)\right) \\
-2 \alpha_{4}\left(\frac{d^{2} w_{s 1}(x)}{d x^{2}}+\frac{\partial^{2} y_{1}(x, t)}{\partial x^{2}}\right)\left(\int_{0}^{1}\left(\frac{\partial y_{1}(x, t)}{\partial x}\right)\left(\frac{d w_{s 1}(x)}{d x}\right) d x\right) \\
-\alpha_{4}\left(\frac{\partial^{2} y_{1}(x, t)}{\partial x^{2}}\right)\left(\int_{0}^{1}\left(\frac{d w_{s 1}(x)}{d x}\right)^{2} d x\right)-\alpha_{4}\left(\frac{\partial^{2} y_{1}(x, t)}{\partial x^{2}}\right)\left(\int_{0}^{1}\left(\frac{\partial y_{1}(x, t)}{\partial x}\right)^{2} d x\right)=0 \\
y_{1}^{\prime}(0, t)=y_{1}^{\prime}(L, t)=0, y_{1}(0, t)=y_{1}(L, t)=0
\end{gathered}
$$




$$
\begin{gathered}
\frac{\alpha_{2}}{\alpha_{1}} \frac{\partial^{2} y_{2}(x, t)}{\partial t^{2}}-\frac{\alpha_{2}}{\alpha_{1}} N\left(\frac{\partial^{2} y_{2}(x, t)}{\partial x^{2}}\right)+\frac{\partial^{4} y_{2}(x, t)}{\partial x^{4}} \\
+\alpha_{2} c_{1}\left(y_{2}(x, t)-y_{1}(x, t)\right)+3 \alpha_{2} c_{3} y_{2}(x, t)\left(w_{s 2}^{2}(x)+w_{s 1}^{2}(x)\right) \\
-3 \alpha_{2} c_{3} y_{1}(x, t)\left(w_{s 2}^{2}(x)+w_{s 1}^{2}(x)\right)-3 \alpha_{2} c_{3} y_{1}(x, t) y_{2}(x, t)\left(y_{1}(x, t)-y_{2}(x, t)\right) \\
-3 \alpha_{2} c_{3}\left(w_{s 1}(x)-w_{s 2}(x)\right)\left(y_{1}^{2}(x, t)+y_{2}^{2}(x, t)\right) \\
-2 \alpha_{2} \alpha_{5} V_{a c} V_{d c}\left(e_{02}+e_{12} y_{2}(x, t)+e_{22} y_{2}^{2}(x, t)+e_{32} y_{2}^{3}(x, t)\right) \cos (\Omega t) \\
-\alpha_{2} \alpha_{5} V_{d c}^{2}\left(e_{12} y_{2}(x, t)+e_{22} y_{2}^{2}(x, t)+e_{32} y_{2}^{3}(x, t)\right) \\
+\frac{\alpha_{2}}{\alpha_{3}} \mu_{1}\left(\frac{\partial y_{2}(x, t)}{\partial t}\right)-6 \alpha_{2} c_{3} y_{1}(x, t) y_{2}(x, t)\left(w_{s 2}(x)-w_{s 1}(x)\right) \\
-6 \alpha_{2} c_{3} w_{s 2}(x) w_{s 1}(x)\left(y_{2}(x, t)-y_{1}(x, t)\right)+\alpha_{2} c_{3}\left(y_{2}^{3}(x, t)-y_{1}^{3}(x, t)\right) \\
-2 \frac{\alpha_{4} \alpha_{2}}{\alpha_{1}}\left(\frac{d^{2} w_{s 2}(x)}{d x^{2}}+\frac{\partial^{2} y_{2}(x, t)}{\partial x^{2}}\right)\left(\int_{0}^{1}\left(\frac{\partial y_{2}(x, t)}{\partial x}\right)\left(\frac{d w_{s 2}(x)}{d x}\right) d x\right) \\
-\frac{\alpha_{4} \alpha_{2}}{\alpha_{1}}\left(\frac{\partial^{2} y_{2}(x, t)}{\partial x^{2}}\right)\left(\int_{0}^{1}\left(\frac{d w_{s 2}(x)}{d x}\right)^{2} d x\right)-\frac{\alpha_{4} \alpha_{2}}{\alpha_{1}}\left(\frac{\partial^{2} y_{2}(x, t)}{\partial x^{2}}\right)\left(\int_{0}^{1}\left(\frac{\partial y_{2}(x, t)}{\partial x}\right)^{2} d x\right)=0
\end{gathered}
$$

where $e_{i 1}(i=0,1,2,3)$ and $e_{j 1}(j=0,1,2,3)$ are the Taylor expansion coefficients of the electric forces applied to the inner and outer layers, respectively. The first subscript represents the coefficient number and the second one stands for the layer number. The terms $w_{s 1}$ and $w_{s 2}$ are the static solutions to the inner and outer layers, respectively, and $\alpha_{i}, i=1-5$ are the ratio constants. In addition, these equations are normalized using $L$ (nanotube length) as a characteristic length and $1 / \omega_{0}$ ( $\omega_{0}$ is the fundamental natural frequency) as a characteristic time. In order to balance the nonlinearity with damping and excitation, the damping and the $\mathrm{AC}$ voltage are considered to be $O\left(\varepsilon^{2}\right)$ and $O\left(\varepsilon^{3}\right)$, respectively. In practical applications of tunable CNT-based nanoresonators, the applied AC voltage is usually much lower than the applied DC voltage to avoid dynamic pullin. Before applying the perturbation method, Eqs. (3a), (3b), (4a), and (4b) are discretized by the Galerkin method using the first vibration mode of a clamped-clamped beam. After implementing the Galerkin method, the dynamic responses, $y_{1}(t)$ and $y_{2}(t)$, are the unknowns in the equations of motions, which are only functions of time. Approximate solutions of the system equations are assumed to be in the following forms:

$$
\begin{aligned}
& y_{1}\left(T_{0}, T_{2}\right)=\varepsilon y_{11}\left(T_{0}, T_{2}\right)+\varepsilon^{2} y_{12}\left(T_{0}, T_{2}\right)+\varepsilon^{3} y_{13}\left(T_{0}, T_{2}\right), \\
& y_{2}\left(T_{0}, T_{2}\right)=\varepsilon y_{21}\left(T_{0}, T_{2}\right)+\varepsilon^{2} y_{22}\left(T_{0}, T_{2}\right)+\varepsilon^{3} y_{23}\left(T_{0}, T_{2}\right),
\end{aligned}
$$

where $T_{0}=t$ and $T_{2}=\varepsilon^{2} t$. In the above equations, $\varepsilon$ is a small dimensionless parameter. Because the secular terms do not appear in $O\left(\varepsilon^{2}\right)$, the slow time $T_{1}$ term is not included in the expansion. Substituting Eqs. (5) and (6) and the polar form of $\cos (\Omega t)$ into the discretized dynamic equations and collecting the coefficients of the same powers of $O(\varepsilon), O\left(\varepsilon^{2}\right)$, and $O\left(\varepsilon^{3}\right)$, one can obtain the solutions to $O(\varepsilon), O\left(\varepsilon^{2}\right)$, and $O\left(\varepsilon^{3}\right)$ successively. After some lengthy derivations, the frequency 
response function of the system can be achieved. Because the coefficients are very sophisticated, it is difficult to present the symbolic notation of the equations. Therefore, the static deflections for $N_{1}=1 e-8, V_{d c}=40$, and the following numerical values are assumed as follows: Young's modulus $E=1 \mathrm{TPa}$, mass density $\rho=1300 \mathrm{~kg} / \mathrm{m}^{3}$, inner tube radius $R_{1}=0.7 \mathrm{~nm}$, outer tube radius $R_{1}=$ $1.4 \mathrm{~nm}$, and the damping coefficients of the inner and outer tubes are the same, $\left(\xi_{1}, \xi_{2}\right)=0.0001$ $\mathrm{Nm}^{-1} \mathrm{~s}$. The length of the DWCNTs is considered as $L=14 \mathrm{~nm}$. The above numerical values are entered into the dynamic equations to obtain the following frequency response equation:

$$
-12 a_{1}^{2}\left(T_{2}\right)\left[3.28 e 16 a_{1}^{2}\left(T_{2}\right)-1.48 e 9 \lambda\right]^{2}+\left(\frac{1}{\gamma}\right)\left[2.3 e-14 a_{1}^{2}\left(T_{2}\right) \bar{\xi}_{1}^{2}(1.65 e 9+1.65 e 9 \gamma)^{2}\right]+1.02 e=V_{a c}^{2},
$$

where $a_{1}$ is the polar amplitude of the amplitude $A_{1}$ of the inner tube displacement and $\lambda$ is the detuning parameter. The terms $\gamma=\bar{\xi}_{1} / \bar{\xi}_{2}$ and $\bar{\xi}_{1}, \bar{\xi}_{2}$ are the normalized damping coefficients of the inner and outer tubes, respectively. To study the stability of the response for the above numerical values of system parameters, it is easier to express $A_{1}$ and $A_{2}$ in Cartesian form and substitute into the frequency response equation to obtain the equations. For each point on the frequency-response curve, the eigenvalues of the Jacobian matrix, obtained from the aforementioned equations, determine the stability of the point. If the frequency of the $\mathrm{AC}$ voltage is close to twice the natural frequency $\left(\Omega=2 \omega_{n}+\lambda \varepsilon^{2}, n=1,2\right)$, it is called a secondary resonance. After some tedious derivations similar to those in the primary resonance, one can finally obtain a very complicated equation for studying the stability behavior of the system.

\section{Stochastic Modeling for DWCNTs Subjected to Electrostatic Actuation}

In this study, the finite element method is utilized to determine the solution to Eqs. (1) and (2). Using the finite element formulation, we can derive the governing matrix equation of the structure after assembly as follows:

$$
[M] \ddot{U}+[C] \dot{U}+S(U)=F
$$

where $[\boldsymbol{M}]$ is the consistent mass matrix, $[\boldsymbol{C}]$ is the damping matrix, $\boldsymbol{U}$ is the displacement vector, $\boldsymbol{F}$ is the external force vector, and $\boldsymbol{S}(\boldsymbol{U})$ is the vector of restoring forces depending on the displacement field. On the basis of Eq. (8), the governing equation of the structure at time $t+\Delta t$ is given by

$$
[\boldsymbol{M}] \ddot{\boldsymbol{U}}^{t+\Delta t}+[\boldsymbol{C}] \dot{\boldsymbol{U}}^{t+\Delta t}+\left[\boldsymbol{K}_{T}^{t}\right] \Delta \boldsymbol{U}=\boldsymbol{F}^{t+\Delta t}-\boldsymbol{S}\left(\boldsymbol{U}^{t}\right)
$$

Here, $\left[\boldsymbol{K}_{T}^{t}\right]$ is the tangent stiffness matrices of the nonlinear system at each time step, which are functions of the displacement field. Equation (9) can be solved by any direct time integration method, even if it is nonlinear. In this study, the Newton-Raphson method and the Newmark scheme are adopted to perform the numerical analysis. By the perturbation technique, the randomly fluctuating Young's modulus of the elasticity $E$ and the mass density $\rho$ can be written as 


$$
E(x)=E^{(0)}[1+\alpha(x)]=E^{(0)}+E^{(0)} \alpha(x), \rho(x)=\rho^{(0)}[1+\beta(x)]=\rho^{(0)}+\rho^{(0)} \beta(x),
$$

where $E^{(0)}$ and $\rho^{(0)}$ are the mean values of the Young's modulus of elasticity and mass density, respectively; $\alpha(x)$ and $\beta(x)$ are random variables with zero mean; and $E^{(0)} \alpha(x)$ and $\rho^{(0)} \beta(x)$ are homogeneous stochastic fields that represent the fluctuation of the Young's modulus of elasticity and mass density to their mean values. Assuming the random variables $\alpha$ and $\beta$ to be uniform within each element, the stochastic nodal displacement vector can be expanded about $\alpha$ and $\beta$ using the Taylor series as

$$
\begin{gathered}
\boldsymbol{U}^{t+\Delta t}=\boldsymbol{U}^{(0) t+\Delta t}+\sum_{i=1}^{N E} \boldsymbol{U}_{i}^{(1 \alpha) t+\Delta t} \alpha_{i}+\sum_{i=1}^{N E} \boldsymbol{U}_{i}^{(1 \beta) t+\Delta t} \beta_{i}+\frac{1}{2} \sum_{i=1}^{N E} \sum_{j=1}^{N E} \boldsymbol{U}_{i j}^{(2 \alpha) t+\Delta t} \alpha_{i} \alpha_{j} \\
+\frac{1}{2} \sum_{i=1}^{N E} \sum_{j=1}^{N E} \boldsymbol{U}_{i j}^{(2 \beta) t+\Delta t} \beta_{i} \beta_{j}+\sum_{i=1}^{N E} \sum_{j=1}^{N E} \boldsymbol{U}_{i j}^{(2 \alpha \beta) t+\Delta t} \alpha_{i} \beta_{j}+\cdots,
\end{gathered}
$$

where the superscript (0) represents the mean value term, both $i$ and $j$ denote the element numbers, $N E$ is the total number of elements constituting the structure, and $\Sigma$ means the summation for all the elements. Similarly, the incremental displacement vector, restoring force vector, and tangent stiffness matrix can be written in the same fashion. By substituting these equations into Eq. (9), applying the perturbation technique with the higher-order terms truncated, and comparing terms of equal order for the random variables $\alpha$ and $\beta$, the zeroth-, first-, and second-order equations for the problem are obtained, respectively. Finally, the eigenvalue problem of the present nonlinear structure system can be stated as

$$
\left[\boldsymbol{K}_{T}^{t}\right]\left\{\Phi^{t}\right\}=\left(\omega^{t}\right)^{2}[\boldsymbol{M}]\left\{\Phi^{t}\right\}
$$

where the values of $\omega$ are the natural frequencies, and $\{\Phi\}$ are the mode shapes. We can solve Eq. (12) easily by any standard algorithm to obtain the eigenvalues and mode shapes. Note that the frequency response function and stability analysis of the nonlinear system can be accomplished in a stochastic sense by using Eq. (12).

\section{Numerical Examples and Discussion}

First, the deterministic analysis of the system is performed. In Fig. 2, the frequency response function near $\omega_{1}$ is presented for specified values of DC and AC voltages by using Eq. (7). This curve is very important for mass sensor applications of DWCNTs, where observing the change in system resonance is an important factor in mass measurement. The circles present the stable solutions and the stars present the unstable solutions. In Fig. 2, stable branches meet unstable branches in saddle-node bifurcation points. The AC voltage-response diagram is presented in Fig. 3. In this figure, the detuning parameter and the $\mathrm{DC}$ voltage are set equal to constant values of $V_{a c}=40 \mathrm{~V}, \lambda=2$. The amplitudes of vibrations are depicted for a range of AC voltages from a very low value to $V_{a c}=2.0 \mathrm{~V}$. As can be seen, there is a multiple valued region for $2 e-0.3 \mathrm{~V}$ $\leq V_{a c} \leq 1.8 \mathrm{~V}$. In the multiple valued regions, stable or unstable solutions are possible depending on the initial conditions and hysteretic response. The points at which the stable and unstable 


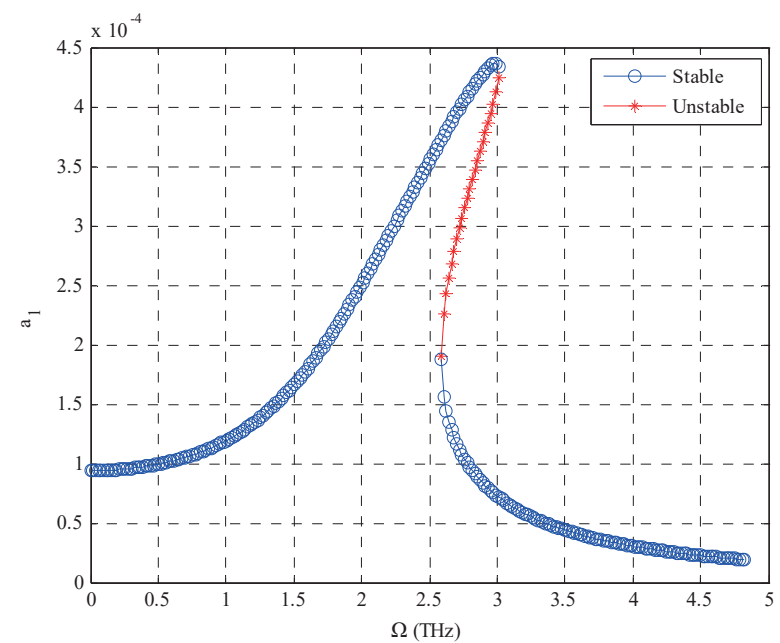

Fig. 2. (Color online) Frequency response function of DWCNTs close to $\omega_{1}$ under primary response condition $\left(V_{d c}=40 \mathrm{~V}, V_{a c}=0.08 \mathrm{~V}\right)$.

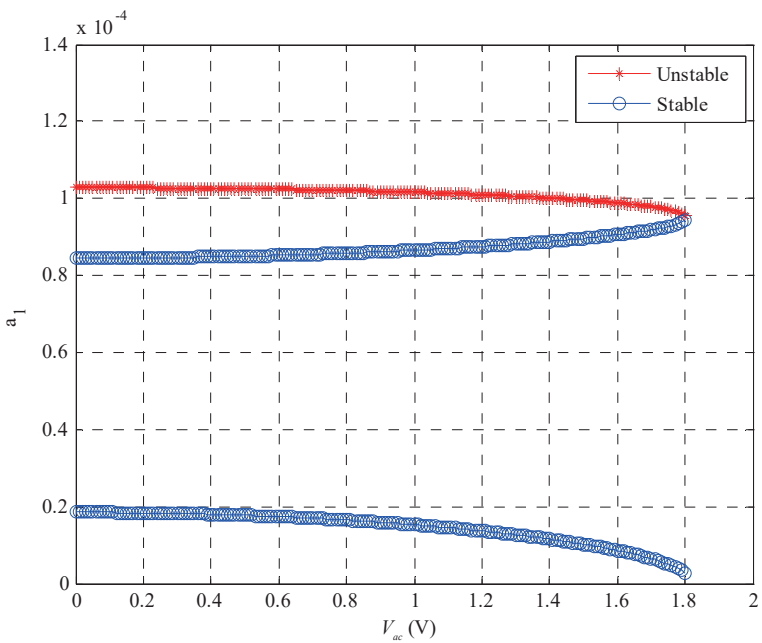

Fig. 3. (Color online) AC voltage-response curve of primary resonance vibration of DWCNTs $\left(V_{d c}=40 \mathrm{~V}\right.$, $\lambda=2)$.

branches meet are saddle-node bifurcation points. Hysteretic behavior happens in DWCNT-based actuators and sensors as well as SWCNT-based systems, ${ }^{(9)}$ but the difference is that in DWCNTs, there are two sets of natural frequencies (coaxial and non-coaxial), where in their neighbors such a hysteretic behavior is detected. In Fig. 4, the stability and instability of the response for the trivial and nontrivial solutions are presented for the case where the DC voltage is equal to $V_{d c}=40 \mathrm{~V}$. This figure shows that for frequencies higher than twice the natural frequency of the DWCNTs, the initial conditions determine if the response is trivial or nontrivial. If the initial conditions are low frequencies, the response is trivial and by decreasing the frequency, it reaches a bifurcation point and jumps to a higher amplitude. By further decreasing the frequency, the response will reach a trivial solution again. The statistical dynamic response of the DWCNTs under electrostatic actuation can be obtained as in Sect. 3, which states the formulations in great detail. Here, the frequency response function and stability analysis are accomplished based on the formulations stated in Sect. 3, particularly Eq. (12). Note that the numerical results in the deterministic analysis based on this study match with those in Ref. 15. In Fig. 5, the frequency response function near $\omega_{1}$ is plotted in the stochastic sense for specified values of DC and AC voltages using Eq. (12), where $U$ is the mean value of the amplitude of the midpoint deflection of the inner tube. To validate this method, Fig. 5 presents the frequency response function of DWCNTs under the primary response condition between this method (circles) and Monte Carlo simulation (diamonds). It can be seen from the figure that they show very good agreement. The AC voltage-response diagram in the stochastic sense is depicted in Fig. 6. In this figure, the detuning parameter and the DC voltage are set equal to constant values of $V_{a c}=40 \mathrm{~V}$ and $\lambda=2$. To study the stability of these points, once again, Eq. (12) is adopted, and the results in the stochastic sense are very similar to those in Fig. 4 in deterministic modeling. 


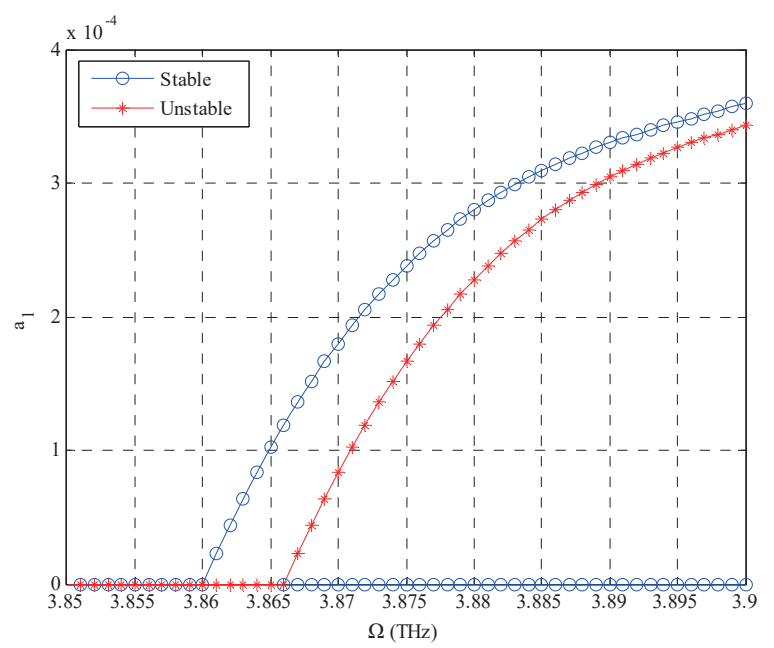

Fig. 4. (Color online) Stability analysis of frequency response function of DWCNTs close to $\omega_{1}$ under secondary response condition $\left(V_{d c}=40 \mathrm{~V}, V_{a c}\right.$ $=0.08 \mathrm{~V})$.

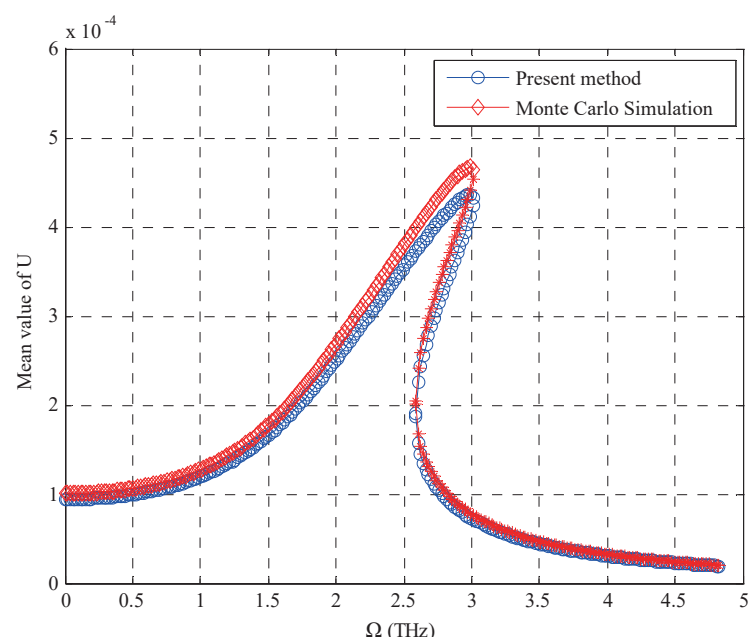

Fig. 5. (Color online) Frequency response function of DWCNTs under primary response condition in stochastic sense $\left(V_{d c}=40 \mathrm{~V}, V_{a c}=0.08 \mathrm{~V}\right)$.

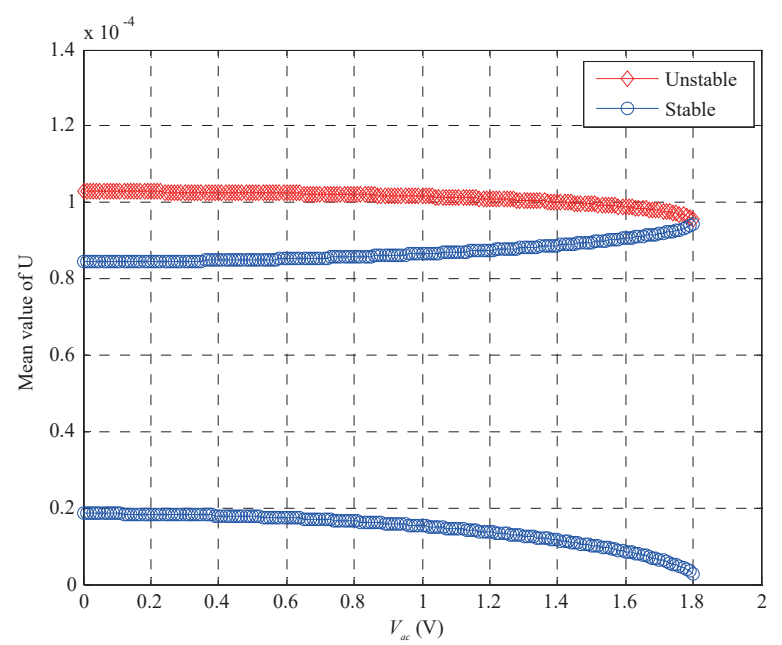

Fig. 6. (Color online) AC voltage-response curve of primary resonance vibration of DWCNTs in stochastic sense $\left(V_{d c}=40 \mathrm{~V}, \lambda=2\right)$.

\section{Conclusions}

The nonlinear forced vibration of DWCNTs is investigated under AC and DC voltages in both deterministic and stochastic senses for two cases: first, the excitation frequency is close to the system's primary resonances, and second, the excitation frequency is close to twice the system's secondary resonances. In the deterministic case, the Galerkin method and perturbation method are adopted to solve the equations of the system. The frequency response function (FRF) of the system is derived for excitations close to the system's natural frequencies and twice their values, and the stability of the response is investigated. The FRFs are then presented for specified values 
of the applied DC and AC voltages. The results show that for the lower values of DC voltage, hardening behavior is observed. Based on frequency-response and amplitude-AC voltage curves, the multivalued regions of the system are detected. The behavior of the system under AC voltage and frequency sweep are also investigated and the amplitude-jumping points of the system are identified for specified values of control parameters. Note that DWCNTs have two sets of mode shapes including coaxial and noncoaxial vibration mode shapes. It can be concluded that the vibrations of SWCNTs and DWCNTs are different in general, but there are some similarities between them that can help scientists understand the behaviors of one case based on the other. In the stochastic case, the finite element method in conjunction with the perturbation method is adopted to investigate the nonlinear forced vibration of the DWCNTs subjected to AC and DC voltages. The FRF of the system is obtained and the stability of the response is investigated. It is concluded that the nonlinear vibration behavior in both deterministic and stochastic cases are very similar; except in the stochastic case, some statistical quantities such as the mean values and standard deviations must be considered and dealt with in the whole process.

\section{Acknowledgments}

This research was partially supported by the Ministry of Science and Technology in Taiwan through Grant No. MOST-103-2221-E-327-011. The author is grateful for the financial support.

\section{References}

1 A. Pantano, D. M. Parks, and M. C. Boyce: J. Mech. Phys. Solids 52 (2004) 789.

2 J. Yoon, C. Q. Ru, and A. Mioduchowski: Phys. Rev. B 66 (2002) 2334021.

3 C. M. Wang, V. B. C. Tan, and Y. Y. Zhang: J. Sound Vibr. 294 (2006) 1060.

4 Y. Zhang, G. Liu, and X. Han: Phys. Lett. A 340 (2005) 258.

5 H. W. C. Postma, I. Kozinsky, A. Husain, and M. L. Roukes: Appl. Phys. Lett. 86 (2005).

6 Y. M. Fu, J. W. Hong, and X. Q. Wang: J. Sound Vib. 296 (2006) 746.

7 K.Y. Xu, X. N. Guo, and C. Q. Ru: J. Appl. Phys. 99 (2006) 064303.

8 M. Dequesnes, Z. Tang, and N. R. Aluru: Trans. ASME 126 (2004) 230.

9 H. M. Ouakad and M. I. Younis: J. Comput. Nonlinear Dyn. 5 (2010) 011009.

10 M. Zamanian, S. E. Khadem, and S. N. Mahmoodi: J. Mech. Eng. Sci. 223 (2009) 329.

11 M. I. Younis and A. H. Nayfeh: Nonlinear Dyn. 31 (2003) 91.

12 E. M. Abdel-Rahman and A. H. Nayfeh: J. Micromech. Microeng. 13 (2003) 491.

13 M. A. Hawwa and H. M. Al-Qahtani: Comp. Mater. Sci. 48 (2010) 140.

14 M. Zamanian, S. E. Khadem, and S. N. Mahmoodi: Smart Mater. Struct. 17 (2008) 0964.

15 A. Hajnayeb and S. E. Khadem: J. Sound Vibr. 331 (2012) 2443. 